This document was prepared ag an account of work sponsored by an agency of the United States Government. Neither the United Siates Government nor the Universily ol California nor any of itheir employees, makes any wartanly, express or implied, or assi mes any legal liability or responsibility for the aceuracy, completenesa, of usefulness of any information. apparalus, product, or process diaclesed or represents that ita use wauld to infringe privately owned rights. Reference herein to any specific commercial products, procest, or "arvice by trade name, trademask, thanufacturer, of otherwise, doet not nectesarily constilute or inaply its erdoruement, recommendation, or tavaring by the Uniled Staies Govemment or the Univertity of Californis. The viewn and opinions of authors expressed herein do not necissarily state or reflect those of the United States Governonknt or the University of Califomis, and thall not be kest for advertising or product endorserent purposes.

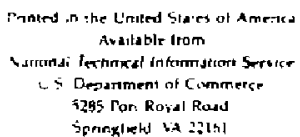

Price

Code

A01

\section{Papercopy Prices}

$\mathrm{A} 02$

A 03

A04

A05

A06

$\mathrm{A} 0 \mathrm{~s}$

Aंग5

A09

\section{Range}

Microfiche

$001-050$

$051-100$

$101-290$

$201-300$

$301-400$

$+01-500$

sor- 6000

601 


\section{A. NUCLEAR DATA CALCULATIONS AND EVALUATIONS}

\section{Charged-particle Evaluations for Fusion Applicacjons (R. M. White)}

The Livermore Nuclear Data Group has for over 10 years maintained an evaluated charged-particle library (ECPL) for $p, d, t,{ }^{3} \mathrm{He}$, and "He particles incldent on most light isotopes through ${ }^{10} \mathrm{O}$. We have initiated a reevaluation of this library for three reasons: (1) to provide new evaluations which include the most recent measurements of important charged-particle reaction cross sectlons for fusion applications; (2) to provide accurate representation of angular distributions, energy distributions, etc., of reaction products for all-particle Monte Carlo type transport calculations; and (3) to provide the most accurate possible cross section data base against which to test effective nucleon-nucleon interattions in our shell model/R-matrix calculational program (see following section). To this end we have comploted new evaluations of the ${ }^{2} \mathrm{H}(d, p){ }^{3} \mathrm{H},{ }^{2} \mathrm{H}(d, n)^{3} \mathrm{He}$, and ${ }^{3} \mathrm{H}(\mathrm{d}, n){ }^{4}$ He reactions from $50 \mathrm{eV}$ to $20 \mathrm{MeV}$. These evaluations include over 60 angular distr.butions for the d-t reaction and

over 30 angular distributions each for the ${ }^{2} H(d, p)$ and ${ }^{2} H(d, n)$ reaction channels. Because the ${ }^{3} \mathrm{H}(\mathrm{d}, n)$ and ${ }^{2} \mathrm{H}(\mathrm{d}, n)$ reactions are widely used as neutron source reactions, there exist several reviews of these reactions in the literacure. For our evaluations of these reactions we have used the work of Liskien and Paulsen, 1 Drosg, ${ }^{2}$ and the recent experimental results of Jarwie, Brown and Hardekopf ${ }^{3}$ as well as the work of Brown, Jarmie and Hale. For the ${ }^{2} \mathrm{H}(\mathrm{d}, \mathrm{p})$ reaction channel, we have obtafned over 70 experimental angular distributions from 10 references which span the energy range of interest. In Fig. 1 we show the integrated results of some of these angular distributions as well as our evaluation of the integrated reaction cross section over the energy range of $100 \mathrm{keV}$ to 1 MeV. The evaluation angular distributions were actually obtained from an evaluated of the Legendre expansion coefficlents derived from fitting the experimental angular distribution data. In the coming year, we intend to begin work on the $d+{ }^{\circ} L I$ reactions channels.

1. Nuclear Data Tables 11, (1973).

2. Nucl. Sci. Eng, 67, (1978) and Atoms and Nuclei 300, (1981).

3. Phys. Rev, C 29, (1984).

4. Phys. Rev. C $35,(1987)$ and private communication. 


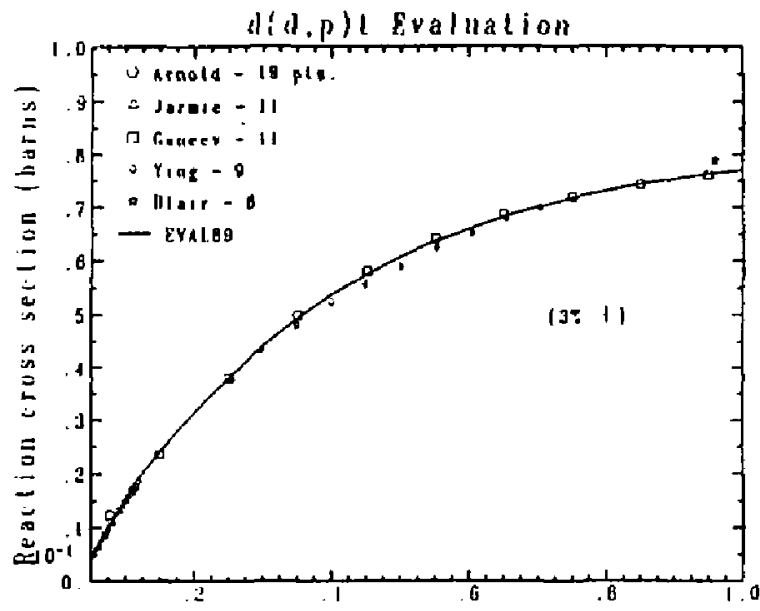

$E_{d} \operatorname{NeV}($ lah)
Fig. 1 Integrated results of exferimental angular distri. butions and our evaluation of the integrated cross section for the ${ }^{2} \mathrm{H}(d, p)^{3} \mathrm{H}$ reacrion over the energy range $100 \mathrm{keV}$ to $20 \mathrm{MeV}$.

2. Extension of the She 12 Model/R-Matrix Approach to AlIow the Calculation of Cross Sections Involving Multinucleon Pacticles $\left(1, e, d, t,{ }^{3} \mathrm{He}, a\right.$, etc $)$ (D. A. Resler and H. D. Knox*)

Last year we reported how the shell model/R-matrix approach was being used to calculate cross sections of interest to the nuclear data community. In the example presented of the $14 \mathrm{~N}(\mathrm{n}, \mathrm{xy})$ production for $14 \mathrm{MeV}$ incident neutrons, we were unable to calculate the entire gamma spectrum since we did no: have the ability to calculate gamma rays resulting from the reactions $\left(n, d^{\prime} \gamma\right),\left(n, t^{\prime} \gamma\right)$, or $\left(n, a^{\prime} \gamma\right)$. The ability to calculate cross sections for reaction channels involving multinucleon particles (i.e., $d, c,{ }^{3} \mathrm{He}, \alpha$, etc.) requires the calculation of multinucleon spectroscopic amplitudes from shell model wavefunctions. In the shell model/R-matrix method of calculating reaction cross sections, a reaction $A\left(k, k^{\prime}\right) B$ can be represented as

$$
\mathrm{A}+\mathrm{k} \rightarrow \mathrm{A}^{\prime} \rightarrow \mathrm{B}+\mathrm{k}^{\prime}
$$

where $A^{\prime}$ is the compound nucleus of the system. In general the size of the reaction cross section is related to how much the states of the compound systi:which can be populated by the entrance channel "look like" A and $k$ coupled together and "look like" $\mathrm{B}$ and $\mathrm{k}$ ' coupled together. As the projectile energy is changed, the compound states populated by the entrance channel change, and the amound that $A$ and $k$ "look like" $A$ ' change as does the amount that $B$ and $k$ " "look like" $A^{\prime}$ changes. Therefore we get a cross section which changes with the projectile energy since we are populating different states in the compound nucleus. 
In terms of the R-matrix theory how much a channel "looks like" the compound system is given by a reduced width amplitude $\gamma$. This can be expressed in terms of a shell model spectroscopic amplitude $s^{1 / 2}$. A spectroscopic amplitude indicates how wuch a given wavefunction "looks like" two other wavefunctions coupled together. In the case of single nuclean transfer, the spectroscopic amplitude is very simple and consists of the product of two factors. The first factor is a constant which depends on the masses of the particleg and some of the quantur numbers. The second factor is simpiy a doubly-reduced matrix element of a compound nucleus wavefunction dotted into the result of creating a single nueleon of the proper quantum numbers onto the target/residual nucleus wavefunction.

In the multinucleon case, the spectroscopic amplitude is more complicated. The first factor is the same as before. In the second factor, the creation of a single nucleon is replaced by the creation of a multinucleon cluster which nov can contain many terms as do the wavefunctions for the compound, target, and residual nucleus. There is also a third factor which is for deterwining how much of the cluster wavefunction is in a relative s-stace since we want the center-of-mass to carr; all the quanta for the cluster. Finally, there can now be more than one cluster wavefunction of the required quantut numbers which can be coupled to the target/residual nucleus wavefunctions. Therefore, the final spectroscopic mplitude is a sumbation over these wavefunctions.

The theoretical details of these multinucleon spectroscopic amplftude calculations have been worked out for use in our m-scheme shell model code CRUNCHER. The new coding is very general--no restrictions on che number of particles in the cluster and no restrictions on the model spaces used. This is believed to be the first time such general coding has been put together for the calculation of multinucleon spectroscopic amplitudes. Extensive resting has been performed and results compared where posstble with those in the literature. With the completicn of this work, all sections of the coding used in our shell model/R-matrix approach to calculating nuclear reaction cross sections are general in that there are no restrictions in which two-body reaction channels can be included. work on the complete calculation of the $14 N(n, x y)$ production is currently underway.

Fohio Universicy, Athens, Ohio

3. Development of an Evaluated Photonuclear Library (S. Warshaw)

To provide complete coupling between neutrons, charged-particles, photons (and electrons) in a new all-particle Monte Garlo transport code being developed at Livermore, the Nuclear Data Group is creating an evaluated photonuclear data library. The data for this library are being collected from three main sources: (1) the NBS 15-volume set of photo-and electro-nuclear cltations and excerpts; (2) systematic scanning of current Concents: and (3) the Dietrich and Berman photoneutron atlas pyblished in 
1988 as Atomic and Nuclear Deta Tables. Vol. 38. This atlas Includes daca for 361 lsotopic and natural targets culled from 64 journal artieles, with a few redundancies. Its chief virtue is that it is the only large collection of moroenergetic photon-induced neutron production reaction 4-pi cross section data known to cover significant photon energy and nuclear mass ranges. From these sources 400 journal references have been collected to dace and photocoples and a cross-referencing index started. If Fig. 2 we show the photoneutrod cross-section for $208 \mathrm{~Pb}$ and 1 ts decomposition into various reaction channel multiplicity subsers. To date data have been processed for ${ }^{3} \mathrm{H},{ }^{1}{ }^{4} \mathrm{He}, 0,7^{01},{ }^{2} \mathrm{H},{ }^{8} \mathrm{Be}, 10,1{ }^{1} \mathrm{~B}$, and ${ }^{200} \mathrm{~Pb}$. The intent is to obtain evaluated photonuclear library flles for each of the Isotopes $(-100)$ curtently in the Ifvermore Evaluated Neutron Data ijlibrary (ENDL).

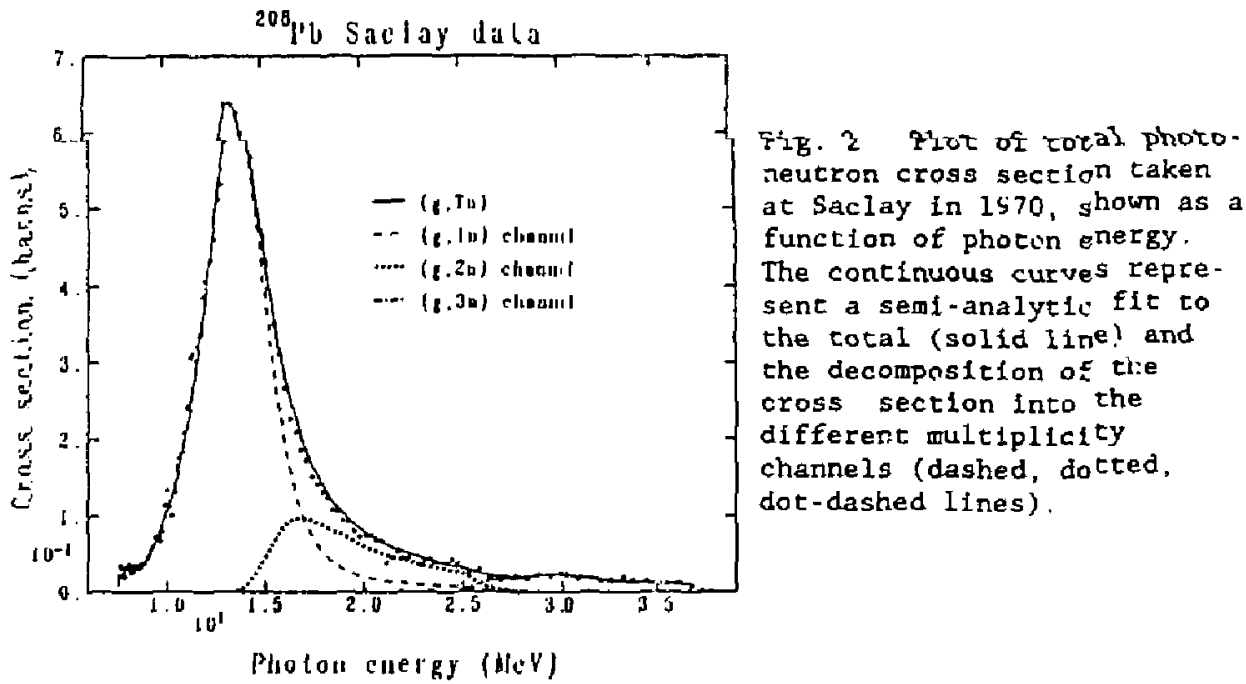

4. Galctization of Trecompoum Eamma - ray spectra Ior Several Materials for Incident Nestrons from 3 to $30 \mathrm{MeV}$ (M. Bland)

The high-energy gamma-ray spectra have been calculated for incident neutron energles from 3 to $30 \mathrm{MeV}$ on natural vanadium, irsn and lead, and on $236,238 \mathrm{U}$ and $23 \mathrm{P}^{\mathrm{P}}$. In this work, only gamma-rays with energies In excess of the neuron olnding energy are caiculated. These gamma-rays originate from a pre-equilibrium decay mechanism. Fig. 3 shows an example for iron and gfves an estimate for the uncertainty in the calculations. For calculations oi light isotopes such as ${ }^{27} \mathrm{Al}$ and $32 S$ the calculations do poorly. Calculated results, the physics employed in the model, and suggested alternoty.re physical models which should work well in the light element regine are sumarized in repdrt UCRL-100324. 


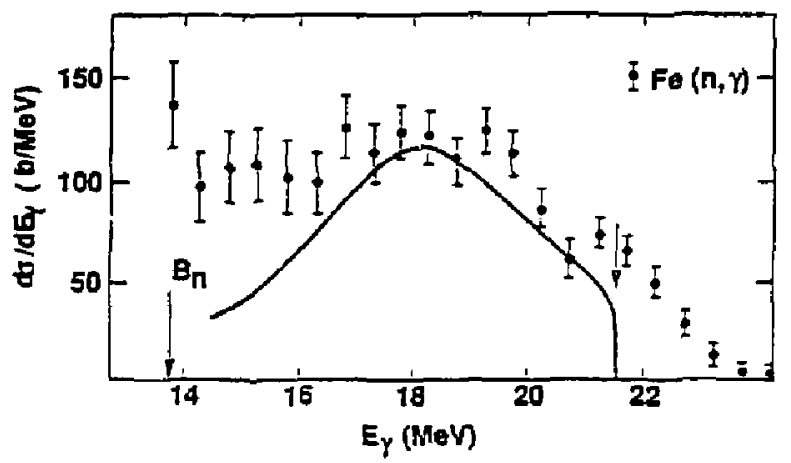

Fig. 3 Experinental and calculated $(n, \gamma)$ spectra for ${ }^{56}$ ge. Experimental results are from Cvelbar, et al., Nucl. Ptys. A310, 40 (1969). The arrow beneath $B_{n}$ represents the energy below which $\gamma$-rays may be emfited following neutron enission. The experimental points extend beyond the maximum $\mathrm{T}$-ray energy due to experimental energy resolution.

5. Improvements of the Precompound Exciton Model in the STAPRE Code (D. Gardner and M. Gardner)

While our present precompound perticle evaporation model adequately describes its total particle emission spectra, and thus its proper contrfbution to total reactions such as $\left(n, n+n^{\circ}\right),(n, 2 n),(n, 3 n)$, etc., the partition of such cross sections among ground and isomeric states may not be as accurately calculated. No formalism of which we are aware properly conserves spin and paricy in precompound calculations. C. Y. Fu, H. Feshbach, G. Reffo, and others have attempted to follow aspects of the physics as the initial excited nucleus proceeds cowards equilfbrium, but there appear to be adjustable paramerers that must be decermined in some ad hoc manner. Our ortginal version of STARRE distributes the precompound contribution at each energy in the daughter nuclei using the same spin distributions in the continum ard ovex the discrete levels as tn the compound case and disregards parity. Fu. Feshbach, Reffo, etc. have suggested the use of an exciton-dependent spin cut-off factor for the particle evaporation from each exciton configuration, as the nucleus approaches equflibrtum. We tmplemented this approash but with some modifications.

For a given exclton state uth $N_{p}$ and $N_{h}-N$ particles and holes, the spin cut-off factor is taken as

$$
\nabla_{\mathrm{P}}^{2}(\mathrm{~N}) \text { - const. } * \mathrm{NA}^{2 / 3} \text {. }
$$

The spin distribution equation is the usual one. Our modifications consis: of making the precompound spin cut-off parameter dependent on the energy in 
each residual nucleus and on its proper normalization. The STAPRE code computes the number of exclton configurations required to "sufficiently" approach equilibrium from a given set of initial conditions. We can show that

which leads to

$$
\sigma_{P}^{2}(\mathrm{~N})-\sigma_{C}^{2}\left(E_{r}\right)
$$

$$
\sigma_{P}^{2}\left(N, E_{r}\right)-\frac{N}{N_{C}} \sigma_{C}^{2}\left(E_{r}\right)
$$

where ${ }_{C} C$ is the particle-hole number of the final exciton configuration and

$$
a_{c}^{2}\left(E_{r}\right)
$$

Is the energy-dependent spin cut-off for the compound nucleus, computed in the usual way. Usually most of the precompound evaporation comes from the first exciton state, resulting in a shift in the population of the daughter nucleus to states of lower spin values. In the subsequent $\gamma$-ray cascade, for those states that do not particle decay. the lower spin isomers and/or ground state will cend to be favored over the higher spin states; the magnitude of this effect will depend on the relacive amouncs of the precompound and compound nucleus processes.

We have also allowed the state denslty and pairing gap values for the exciton model (Including that of the residual nuclel) to be spectfied as input parameters. In many precompound models, the matrix element, $\left|\mathrm{H}^{2}\right|$, for the residual interaction, is taken to be excitation-energy and mass dependent, as $\left|\mathrm{H}^{2}\right|-\mathrm{k}_{1} \mathrm{~A}^{-3} E^{-1}$. The new input option allows $\left|\mathrm{M}^{2}\right|$ to be related to the Fermi gas level density parameter if desired. We are now conducting senstivicy studies to determine the importance of the above exciton model changes.

6. Eyaluated Set of Neutron Cross Sections for 20 Ne from IDA Modeling Calculations (M. H. MacGregor, R. J. Howertor, and G. Reffo*)

As reported last yesr, the Iluclear Data Group has implemented the extensive system of nuclear modeling codes (IDA) developed by G. Reffo. These codes were used together with the very limited experimental data to obtain a complete set of evaluated neutron cross sections for $2{ }^{\circ} \mathrm{Ne}$ from 0 to $30 \mathrm{MeV}$. The IDA codes are based or the Hauser-Feshbach statistical model formalism with width Eluctuartons included. In the $20^{\circ} \mathrm{Ne}$ evaluation, the IDA calculations, modifled in some places to agree with experimental data, were used from 1 to $30 \mathrm{MeV}$. Below $1 \mathrm{MeV}$, cross sections were obtained from experimental neutron resonance parameters. The Livermore post-processing codes ALPHA and OHEGA were used to check energy balances for all of the reactions individually, so that the final avaluated data set accurately conserves energy. Figure 4 shows a comparison between the theotetical IDA $(n, a)$ cross section curve and experimental deta. As 
can be seen, the theoretical curve gives the proper chreshold behavior and a good fit to the deta up to $4 \mathrm{MeV}$. Above $4 \mathrm{MeV}$, the evaluated data set was modifled to macch experiment.

*ENEA, Bologna, Italy

Fig. \& Comparison between the calculated $20 \mathrm{Ne}(n, \alpha)$ cross section using IDA and the experimental data of C. H. Johnson, et al. Phys. Rev. 82,117 (1951). and chose of R. J. Bell, et al. Nuc]. Phys. 14, 270 (1959). The calcularion was subse. quently altered in the evaluation to fit the experimental daca.

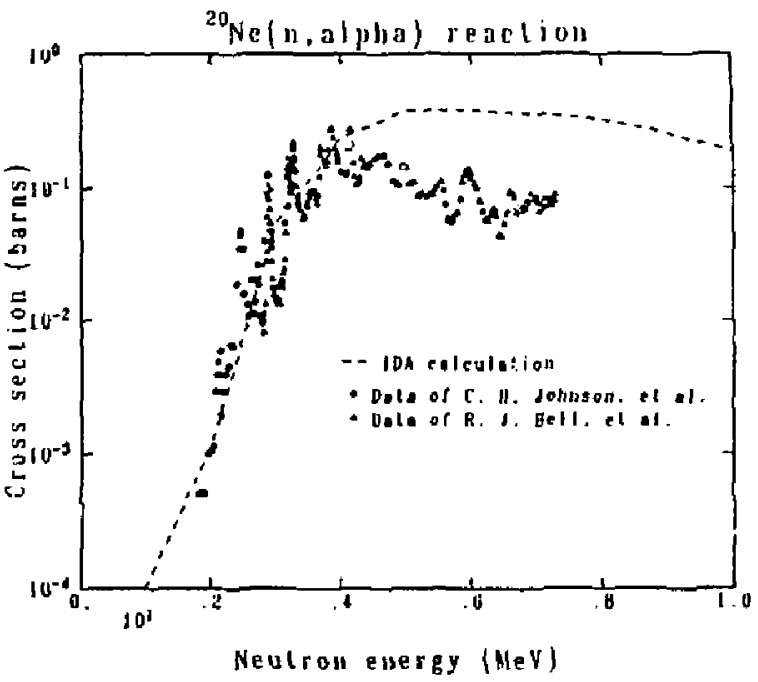

7. Measulement and Analys15 of Neutron and Gama-Ray Spectra Leaking from Several Materials Centrally Pulsed by $14-\mathrm{MeV}$ Neutrons

(E. Goldberg, L. F. Hansen, T. T. Komoto, B. A. Pohl,

R. J. Howerton, R. E. Dye, W. E. Warren, and E. F. Plechaty)

Nonte Carlo calculational analysis of several integral measurements have been completed with two different data bases, ENDL and ENDF/B-V. The experiments utilized spherical assemblies, pulsed centrally with $14-\mathrm{MeV}$ neutrons. The measured neutron and gamma-ray leakage spectra were compared to the spectra calculated with TART and SANDYL. Preparation of the ENDF/B-V data base for use in TART proved to be a formidable task. Figures $5 a$ and $5 b$ showing the neutron time-of-flight and recoil electron spectra for aluminum, typlfy the findings of the study. Both calculated neutron spectra agree reasonably well with the meazurement. Howsver, we see the EADF/B-V-based recoil electron spectrum to be below the experimental data while the ENDLbased spectrum is in close proximity to the experimental points.

We have chosen as a measure of gamma-ray energy deposited in the NE-213 derector the quanticy $\Sigma_{n_{i}} c_{1}$ where $n_{1}$ is the number of counts in channel $c_{1}$. Ratios of these summations, evaluaced with the findings from the pulsed-sphere experiments, have been applied to TART calculations of simple one-zone, one-dimensional spheres. These results may be used by the 
general audience as benchmark exercises. Table 1 lists our findings. He see immediately that silicon generates the largest gamma-ray element. If we rely wpon the ENDL base we find that $2.008 \mathrm{HeV} / \mathrm{n}$ leaks irom the centraliy-pulsed sphere, while rellance on the ENIF/B-V bese leaks $2.268 \mathrm{MeV} / \mathrm{n}$. The maximum leakage is found to occur when $\rho \mathrm{R}=35 \mathrm{~g} / \mathrm{cm}^{2}$.

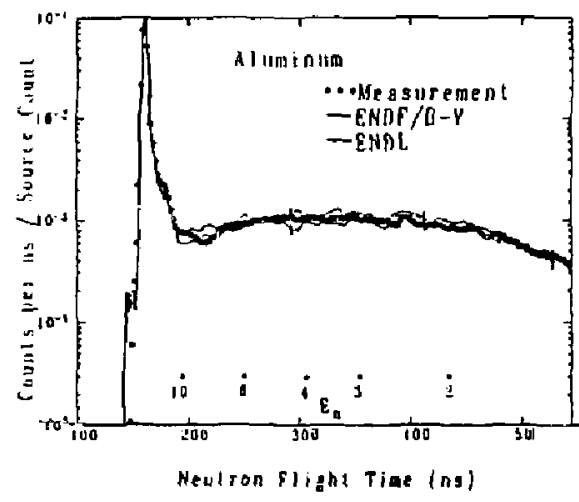

Fig. 5 a

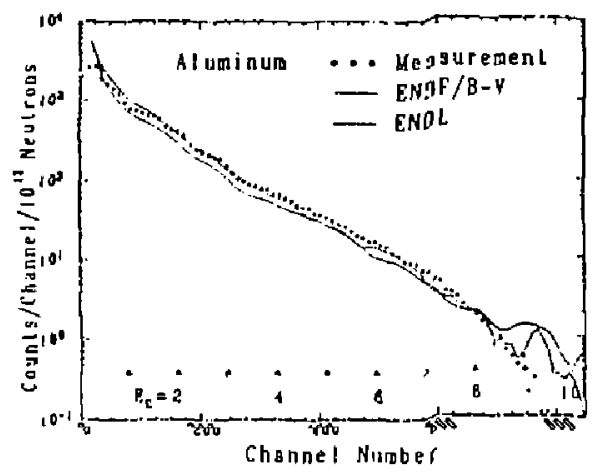

Fig. $5 b$

Figures $5 a$ and $5 b$, showing the neutzon time-of-flight and recol 1 electron spectra for alumintm, typify the findings of the study.

Table 1. Gama Ray MeV Leaked/14-MeV Neutron, Normalized to Pulsed Sphere Experiments

\begin{tabular}{|c|c|c|c|c|c|}
\hline & $(\gamma$ & $\mathrm{lev} / \mathrm{n})$ & $\mathrm{HeV}$ & D) $/(C / E)$ & $(\rho R) \mathrm{Ma}$ \\
\hline Material & ENDL & ENDF/B-V & ENDL & ENDF $/ B-V$ & $9 \mathrm{~g} / \mathrm{ct}$ \\
\hline C & 0.90 & 0.85 & 0.99 & 1.03 & 40 \\
\hline $\mathbf{N}$ & 1.35 & 1.14 & 1.38 & 1.46 & 40 \\
\hline $\mathrm{H}_{2} \mathrm{O}$ & 0.43 & 0.7 & 0.81 & 0.87 & \\
\hline $\mathrm{C}_{2}^{2} \mathrm{~F}_{4}$ & 0.98 & 0.61 & 0.72 & 0.69 & 2 \\
\hline AI & 1.97 & 1.58 & 1.82 & 1.98 & 35 \\
\hline Si & 2.15 & 1.67 & 2.00 & 2.26 & 35 \\
\hline$T I$ & 1.44 & 1.53 & 1.84 & 1,68 & 45 \\
\hline $\boldsymbol{F e}$ & 1.49 & 1.5 & 1.37 & 1.49 & 0 \\
\hline $\mathrm{Cu}$ & 0.89 & 0.99 & 0.91 & 0.93 & \\
\hline Ta & 0.51 & 0.43 & 0.56 & 0.55 & \\
\hline$W$ & 0.42 & 0.39 & 0.52 & 0.48 & \\
\hline $\mathrm{Au}$ & 0.33 & & 0.35 & & \\
\hline 232 & 0.35 & 0.29 & 0.30 & 0.31 & \\
\hline 232 Th & 0.34 & 0.29 & 0.35 & 0.34 & \\
\hline & 0.38 & 0.32 & 0.37 & 0.38 & \\
\hline
\end{tabular}


Phenomenological optical model calculatiuns have been carried aut which gite satisfactory fits to the total and differential neutron cross sections for nuclei in the actinide region ( $T$ h-Pu) for incident neutron energifs above $10 \mathrm{MeV}$. These calculations were periormed by mudifylng one of Ohio University's global spherical optical potentials to include the nonsphericlty of chese target nuele1. The modifled potential depends on the deformation paramecer, $B$, which is determinec by experimental data, e.g., Coulomb excltation to low-lying rotational states. With this modified potentisl, cross sections calculated fur actinide nuclai where no experimental data exists can be carried out with sone confidence.

1 more sophisticated treatment of neut:0, interactions with actinide nurlel involves carry Ing out coupled-channt 1 calculations where the rotational and vibrationa? degrees of freedom of the target nuclei can be taken 1 nt.o account more realistically. Preininary coupled-channel calculations with the codi: ECIS have been performed on the Livermore Nuclear Data croup's Sun computer system. To date, equivilent calculations performed with ECIS on the Cray and the Sun computers are In good agreement.

*Pennsylvania State University

9. Further Developments i- the Study of our Neutron-Induced Cross-Section Library for Isotopes of ir idium, (M. Gardner and D. Gardner)

As we mentioned last year in this report, iridiun is icen used as a detector to measure neutron fluences in underground nuclear cests. I $r_{2}$ order to fully exploit the potential of this detector, we have completed che calculation of a preliminary set of cross sections peimarily for sensitivity stidies. We computed 228 exaitation functions for partial and cotal reactions among 22 ground and isomertc states of 10 iridium isotopes, fron. 107 Ir to $19{ }^{\circ} \mathrm{Ir}$. The principal problems we'va encountered in our calculations lie in the fact that our radlochemica? diagnostic measurements involve fither single Isomers or sums of the ground state plus one or more isomeric populations lance portions of some of the cross sections enc up in long-Iived iscmeric states) and that the discrete-level information req'ired. in our computations, as well as most of the experimental reaction cross section daca are presentiy incomplete and/or uncercan. Ir order to preperly calculate the ground- and isomerle-state populations correc $-1 y$, complete set of reliable discrete nuclear levels and their multipole branchings must be avaflable for each Ir isotope. Gased on our Initlal calculaticns, we have been able to point out problems in the level information, particularly for the odd-odt Ir isotopes. Much new experimental and theoretical work is now under way by other members of our Nuclear Chemistzy Df.vision, primarily on the 1sotopes $191,192.103,204 \mathrm{IT}$. 
We have undertaken the evaluation of the new data and information as they become available. These Include the analysis of new average resonance capture (ARC) data from $2-\mathrm{keV}$ neutrons on ${ }^{101}$ Ir taken by $R$. $W$. Hoff, calculated gamma-ray production information from $\left(n, n^{\prime}\right)$ reactions on 103I:, and statistioal analyses on spin and parfty distributions, spin cut off parameter: and level densities of new theoretical discrete level set.s. We have designed, and with G. Lursen, essentially completed a couputer program which will search for typographlcal and physics errors in large level sets and their multipole branches, end which will also calculate E1, MI, and E2 transitions among single-perticle intrinsic states and anong rotational bandheads with $K$-quantum number restrictions, if desired.

Additionally we are carrying out sensitivity studies on the $201,193 \operatorname{Ir}(\pi, x \pi)$ cIoss-section calculations to evaluate the improvements that we made in the procompound exciton model in our verston of the STAPRE nuclear reaciion code.

\section{R-Matrix Code Improvement (0. A. Resler)}

Last year we reported that the Ohio Unfversity R-matrix code was made operational on our Sun computer network. Extensive testing has been performed to verify the correct operation of the code. During an investigation of the low-energy d+c reaction, we disccvered a problem with the calculation of the Coulomb wavefunctions since the original coding was not valid in this energy region. The caiculation of the Coulomb wavefuntions has been upgraded to use the coding of Bardin et al. I which is valid for the entfre $(\eta, p)$ plane. Publfshed R-matrix calculations from Jarmie, et al. ${ }^{2}$ and Brown, et al. ${ }^{3}$ for the low-energy d+t reaction were extremely beneficial in verifying the correct operation of our coding as we have duplicaced their very low-energy results to several significant digies.

1. C. Bardin, et al. Computer Physics Communications 3,73 (1972).

2. N. Jarmie, R. E. Brown, and R. A. Hardekopf, Phys. Rev. C 29, 2031 (I984).

3. R. E. Brown, N. Jarmie, and G. M. Hale, Phys. Rev. C 35, 1999 (1987).

11. Computer Network for Nuclear Modeling Calculations (D. A. Resler, T. T. Komoto, and J. D. McGowan)

The Nuclear Data Group's network of Sun Microsystem computers, used for our nuclear modeing calculations, has been upgraded during the past year. The two Sun $3 / 260$ servers have been replaced with Sun $4 / 260$ s. Th1s upgrade gives us a factor of two increase in our calculational capability. In addition we have increased the memory of both machines for a total of 64 Mbytes on one and 16 Mbytes on the other. Many of the shell model/R-ratrix calculations of nuclear reaction cross sections have required the full 64 Mbytes of the larger machine. We have also added a fast $8 \mathrm{~mm}$ tape system to our network for backing up our 3 Gbytes of disk storage. 
12. Uprrades to the Shell Model Code CRUNCHER (D. A. Resler and S. M. Grimes*)

Several major edvances have been made to the shell model code CRUNCHER. The calculation of nuclear reaction cross sections has required the use of very large model spaces which in turn has required a large amount of computer resources. In a typical application of the shell model/R-matrix approach, the shell model part of the calculations can easily require over 958 of the totel time. Therefore, it was worthwile to better optimize the coding for these large problems. A major improvement was the use of multiparticle Hamiltonfans for those problems considered "small" in terms of the computer size. Today a "small" problem can be a 2 the model space description of a p-shell nucleus. Our previous rethod was to keep the Hamiltonian expressed in a two-body form. Now we can use the fast but large multiparticle Hamiltonian on the small problems and still use the slower but smaller two-body Hamiltonian for the larger problems. The use of the multiparticle Hamiltonian has resulted in two orders of magnitude improvement in the execution speed for these problems.

Improvements in the Ohio University version of the code have been incorporated finto the LINL version. Part of the effort at Ohio has been to improve the "unstable basis" portion of the code. This is very important for the calculation of level densities, the first scages in a diagonalization using a two-body Hamiltonian, and in the generation of a mulciparticle Hamiltonian From the two-body Hamiltonian.

*Ohio University, Athens, Ohio

B. NUCLEAR DATA MEASUREMENTS

1. Proton and Deuteron Excitation Functions for ${ }^{151} \mathrm{Eu}$ and

$153 E_{u}$ (H. I. West, Jr., R. G. Lanier, M. G. Mustafa, R. N.

Nuckolls, J. Frehaut*, A. Adan*, and C. A. Phills*)

We have measured the prcton and deutexon excitation functions

for ${ }^{151} \mathrm{Eu}$ and ${ }^{153} \mathrm{Eu}$ for the reactions $151,153 \mathrm{Eu}(\mathrm{p}, \mathrm{n})^{151,153} \mathrm{Gd}$,

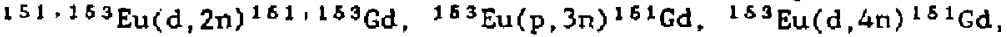

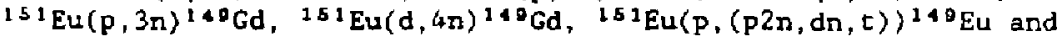
$151 \mathrm{Eu}\left(\mathrm{d},\left(\mathrm{p}^{3} \mathrm{n}, \mathrm{d} 2 \mathrm{n}, \mathrm{tn}\right)\right)^{140} \mathrm{Eu}$. These excication functions are shown in Figures 1-6. In addition a new half-life of $9.282 \pm 0.008 d$ was obcained for $140 \mathrm{Ca}$.

We used the stacked foll method for obtaining the data. Europium oxidizes rapidly in air bursting into flame at $150-180^{\circ} \mathrm{C}$ thus posing a target problem. We solved this problem by mixing $\mathrm{Eu}_{2} \mathrm{O}_{3}$ with a resin which when polymerized is the equivalent of Kapton (a Dupont trade name). 


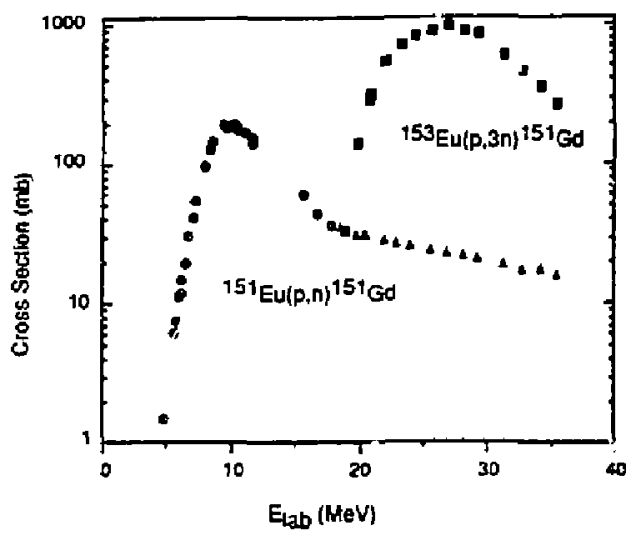

F1g. I

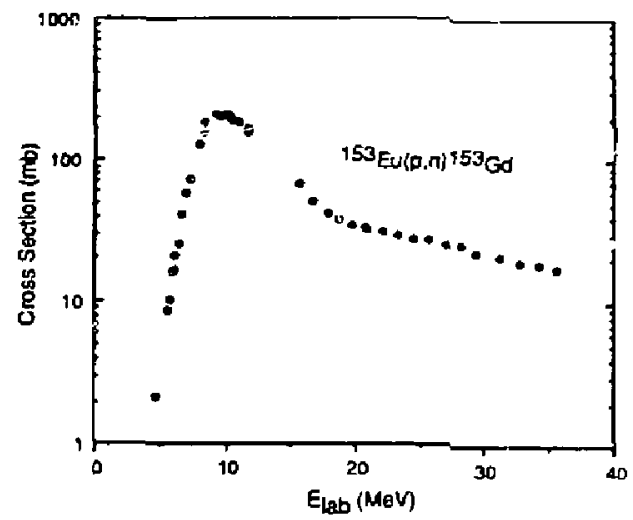

Fig. 1

The foils held up well under irradiation. Corrections were made for the charged-particle energy loss in the target foils in the rapidly varying region of the excitation functions. We plan to use a similar mechod in measuring the cross sections for lodine and bromine.

The measurements at less than $12 \mathrm{MeV}$ were made using the an de Graaff at Cencre d'Etudes Bruyers-le-Chatel, France. The measureme its at

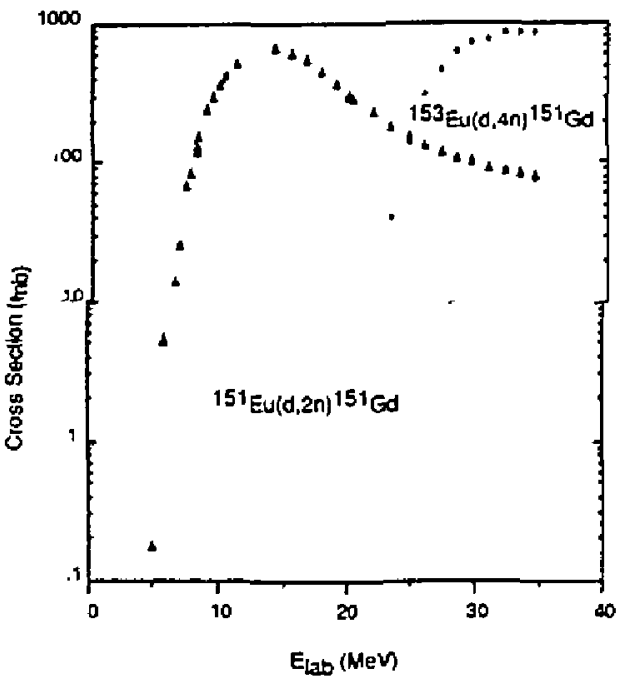

F18. 3

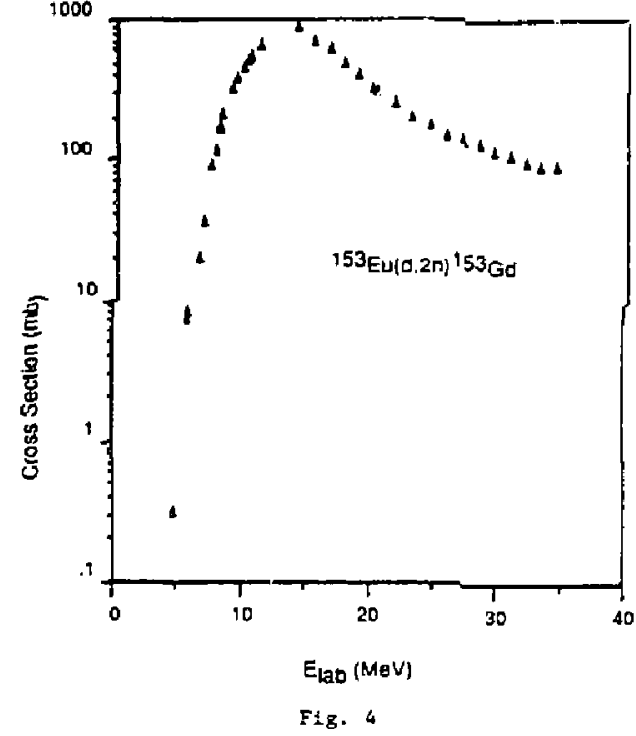




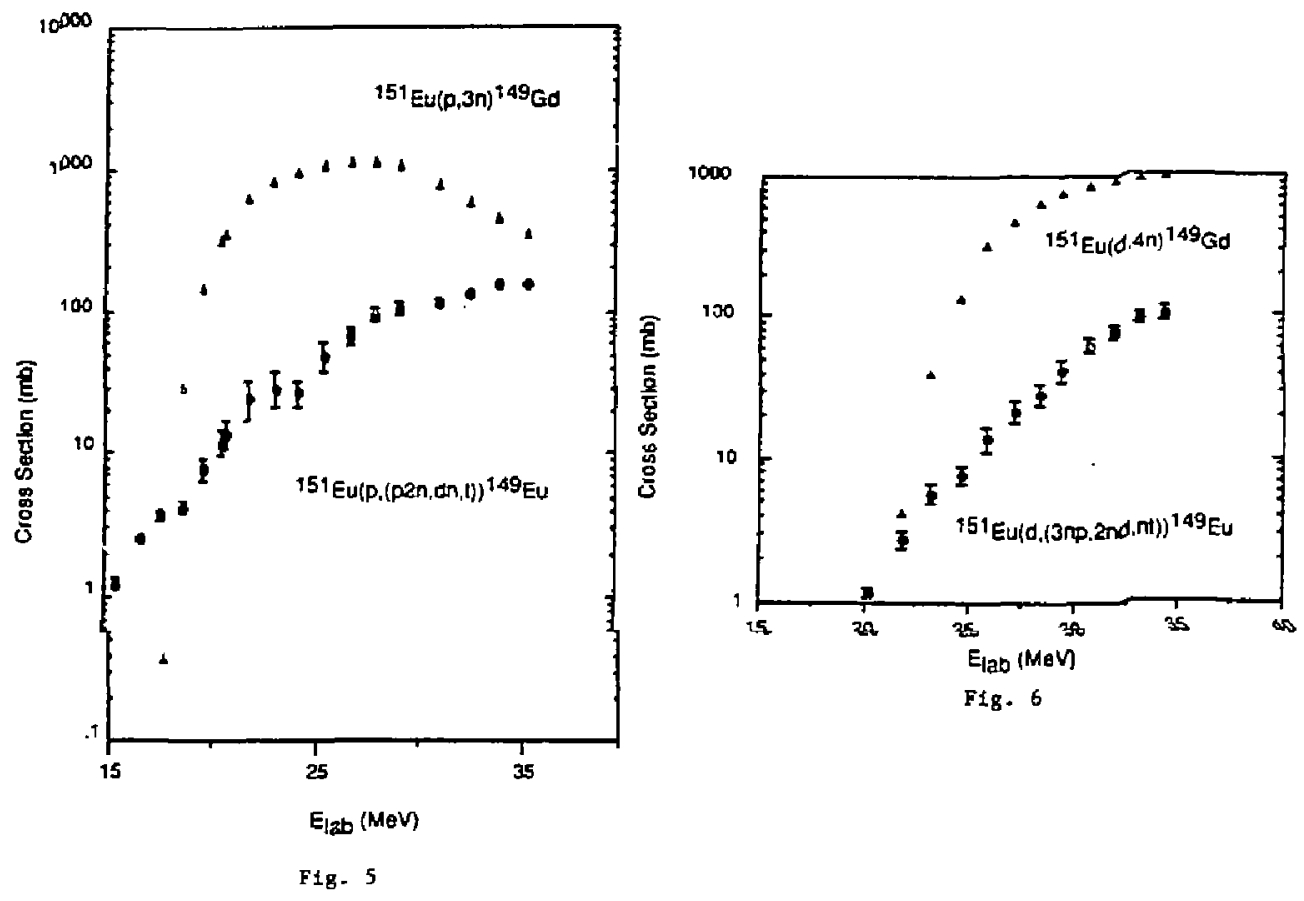

the higher energies were made using the cyclotron at the Crocker Nuclear Laporatory, University of Calffornia at Davis. Although the data appeared to be of high quality we plan to make complementary measurements ir the near future at 12 to $20 \mathrm{MeV}$ using the Van de Graaff presently being installed at LINL.

We have been modeling the data by means of the statistical model cofle STAPRE. However, one aspect of the data requires coupled-channel calculations. We find an interesting feature in the $151,153 \mathrm{Eu}(p, n)^{\text {ts }}, 153 \mathrm{Gd}$ and $131.133 \mathrm{Eu}(d, 2 n)^{151}+153 \mathrm{Gd}$ data; $\sigma(153)$ is enhanced at low energies relative to $\sigma(151)$. Results for (1,n) data are shown in Fig. 7 . We note that the results obtained using a ipherical optical potential are in poor agreement with the daca. However, the results of a coupled-channel calculation, taking into account the fact that for ${ }^{151} \mathrm{Eu}$ the shape paranter $\beta_{2}=0.13$ and for ${ }^{153} \mathrm{E} 1 \hat{\beta}_{2}=0.28$, are in reasonable agreement with the daca at less than $10 \mathrm{MeV}$ where the calculations are expected to be valid. ilearly. we have found a marked shape effect. The results have beer prepared for submission to Physics Le:ters.

*Centre d’Etudes Bruyeres-le-Chare:, France 


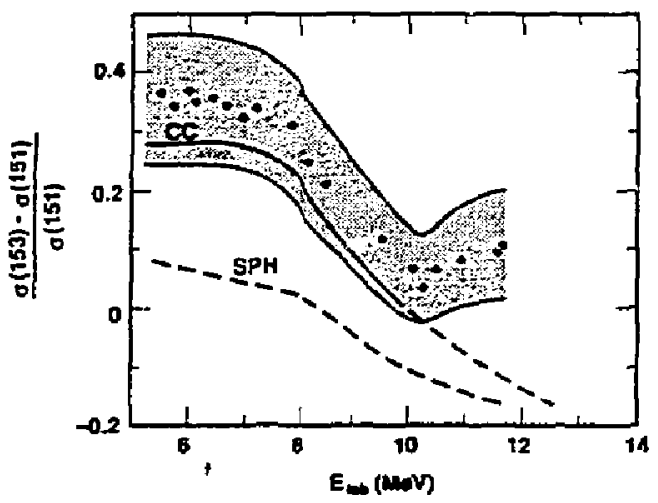

Fig. 7 Evidence of the effects of nuclear shape on reaction cross fections. The band shows the range that the data could take due to uncertainties in the Iy's ued in calculating the cross sections. SPH shows results abtained isting a spherical optical potential and $C C$ shows results for a coupled channel calculation.

2. Comparative Study of Elastic Ne:atron and Proton Scattering and Analyzing Powers using Microscopic Optical Model Potentials in the Energy Range B-26 MeV. (L.F. Hansen, F.S. Dietrich, and R. L. Walter*)

Differential cross sectias and analyzing powars for neutrons ejastically scattered from $10,11 \mathrm{~B},{ }^{28} \mathrm{SI}, 5+\mathrm{Fe}$ and ${ }^{88} \mathrm{Ni}$ messured by trie TUNL (B-17MeV) and Ohio (20.26 MeV) groups and the Eindhoven proton dita $(17-25 \mathrm{MeV})$ are compared with the predictions of microscople optical model calculations using the JUH' and Yamagucht ec al. ${ }^{2}$ potenclals. Tte fits to the differential cross sections and analyzing powers (protons ard neutrons) obtalned with these two potentials are reasonably good using orly three parameters, $\lambda_{\mathrm{V}}, \lambda_{\mathrm{W}}$ and $\lambda_{\text {So, }}$, (normalizing constants for the real.

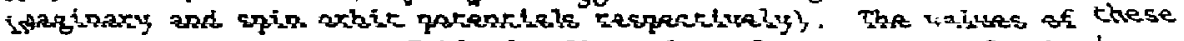
parameters are given in Table 1 . The value of $\lambda_{R}$ is unity for both neutrons and protons, which reduces the number of parameters to two over the energy and mass ranges. Fig. 1-10 fllustrate the quality of the fits to the data for ${ }^{58} \mathrm{NI}$ as function of neurron and proton energy.

* Physics Department, Duke Universiry.

1 J.P. Jeukenne, A. Lejeune, and C. Mahaux, Phys. Rev. C16, 80 (1977); M3Y spin-orb1:, Bertsch et al., Nucl. Phys. A284, 399 (1977).

$2_{N}$. Yamaguchi et al., Prog. Theor. Phys, (Japan) 70,459 (1983). 
(niso $(n, n)$

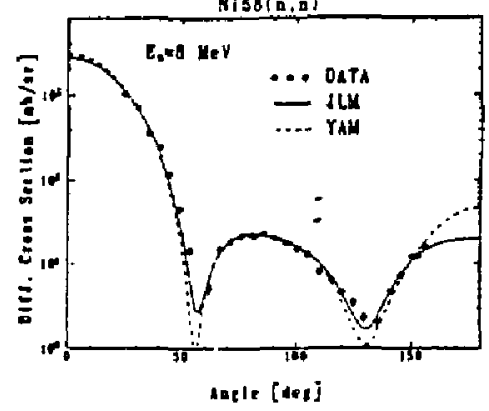

Nisalg,a)

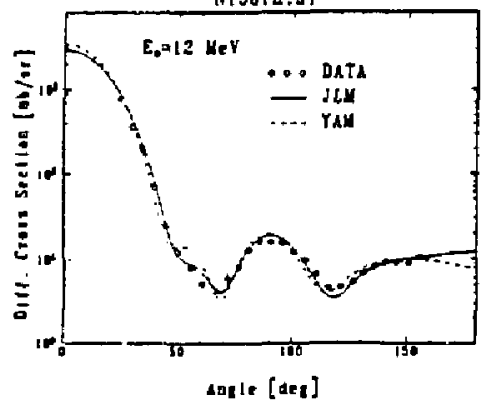

Nisara.gl

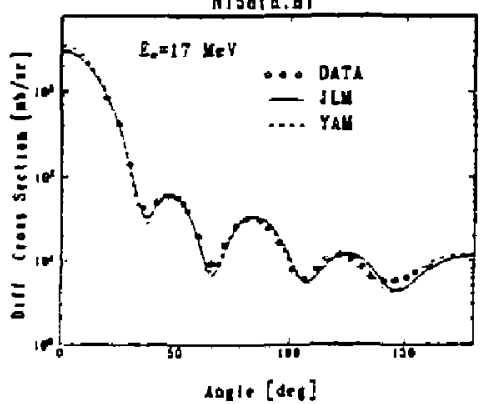

Miss(a.s)

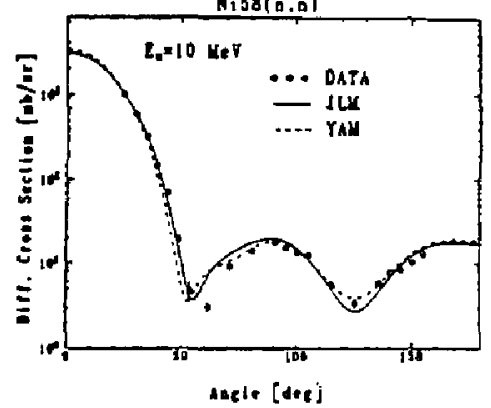

Niso(a,a)

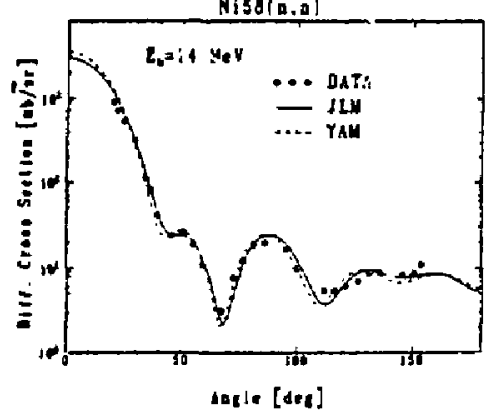

Nisota.a

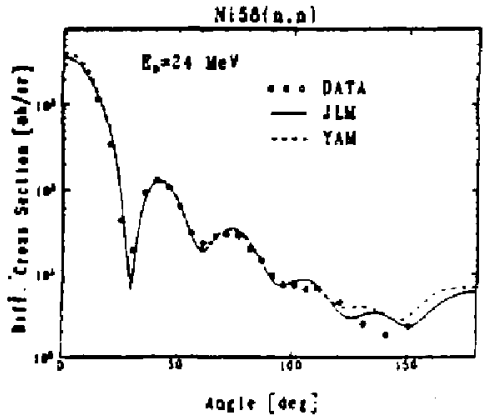

Fig. 8 Neutron elastic differential cross sections for ${ }^{58}{ }^{\mathrm{Ni}}$. Cilculations carried out with the microscopic OMP of Jeukenne-Lejuene Mahaux (JIM: solid lines) and that of Yamaguchi, et al. (YAM: dashea lines). 

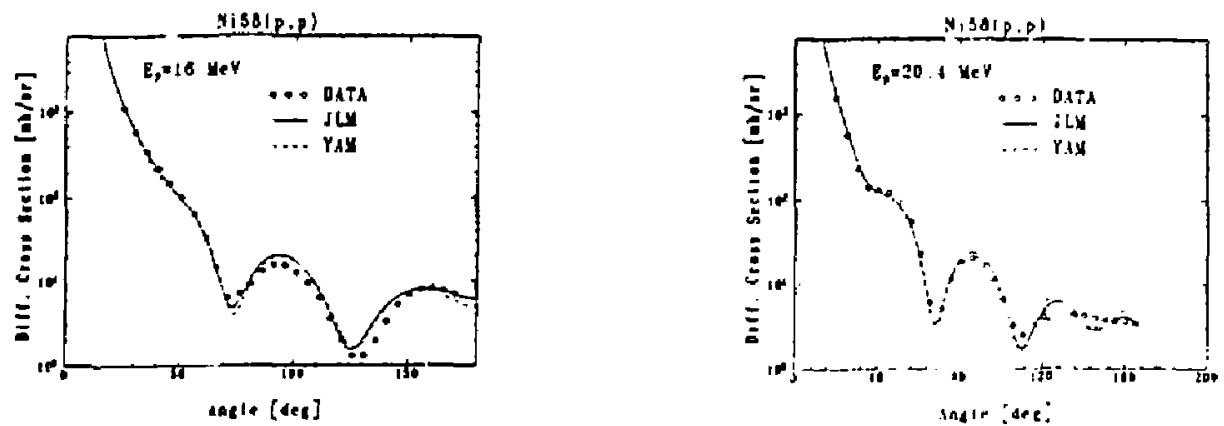

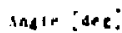

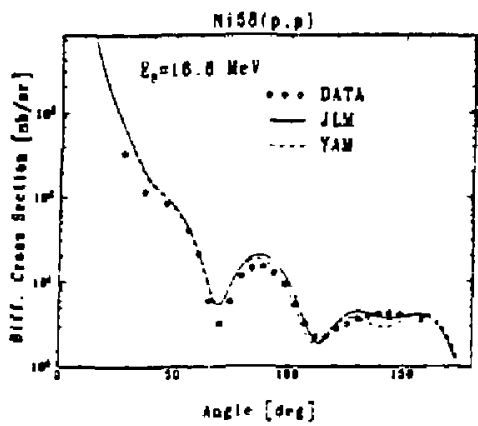

Fig. 9 Proton elastic differential cross sections for ${ }^{58} \mathrm{~N} 1$. Calculations carried out with the microscopic OMP of Jeukenne-Lejeune-Mahaux (JIM: sulid lines) and that of Yamaguchi, et al. (YAM: dashed lines).

Table 1. Values of the three parameters used in the microscopic optical model calculations for all the nuclei and energies included in this report.

\begin{tabular}{|c|c|c|c|c|}
\hline$O M P$ & & $\lambda_{V}$ & $\lambda_{W}$ & ${ }^{\lambda} \mathrm{S} .0$. \\
\hline \multirow{2}{*}{$J I M$} & $n$ & $1.00 \pm 0.04$ & $0.94 \pm 0.15$ & $1.31 \pm 0.05$ \\
\hline & $P$ & $1.03 \pm 0.06$ & $0.91 \pm 0.10$ & $1.52 \pm 0.05$ \\
\hline \multirow{2}{*}{ YNM } & $n$ & $1.00 \pm 0.06$ & $0.70 \pm 0.10$ & $1.45 \pm 0.06$ \\
\hline & $P$ & $1.02 \pm 0.03$ & $0.66 \pm 0.07$ & $1.54 \pm 0.10$ \\
\hline
\end{tabular}



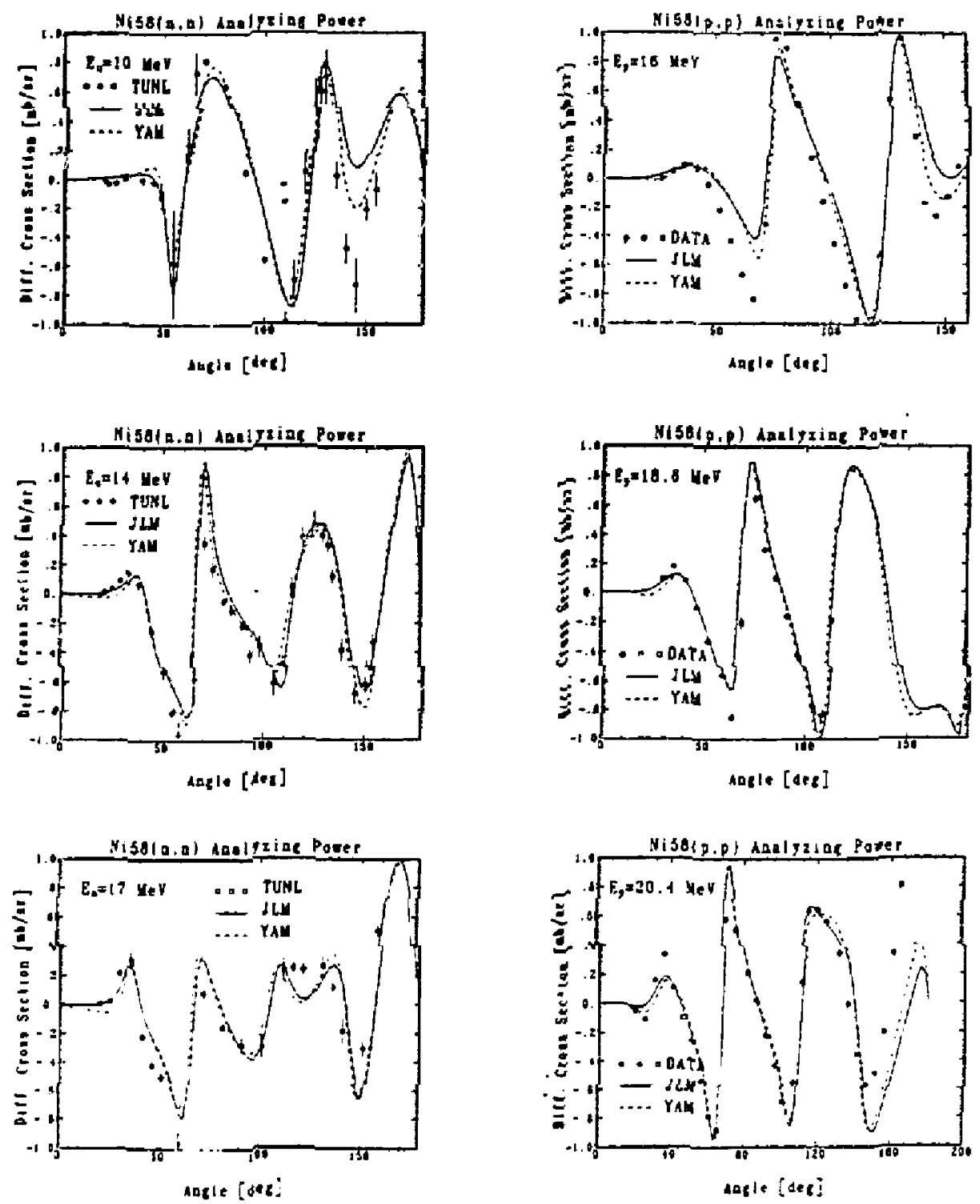

Fig. 10 Ariifzing powers for neutron (left side) and proton (rignt side) elastic scattering from ${ }^{58} \mathrm{Ni}$. Calculations carried uw with the microscopic OMP of Jeukenne-Lejeune-Mahaux (JLM: $s(1$ id lines) and that of Yamaguchi. et al. (YAM: dashed lines). 